
\title{
$\begin{array}{ll}\text { Research Square } & \begin{array}{l}\text { Preprints are preliminary reports that have not undergone peer review. } \\ \text { They should not be considered conclusive, used to inform clinical practice, } \\ \text { or referenced by the media as validated information. }\end{array}\end{array}$
}

\section{The early impacts of COVID-19 on unpaid carers of people living with dementia: Part II of a mixed-methods systematic review}

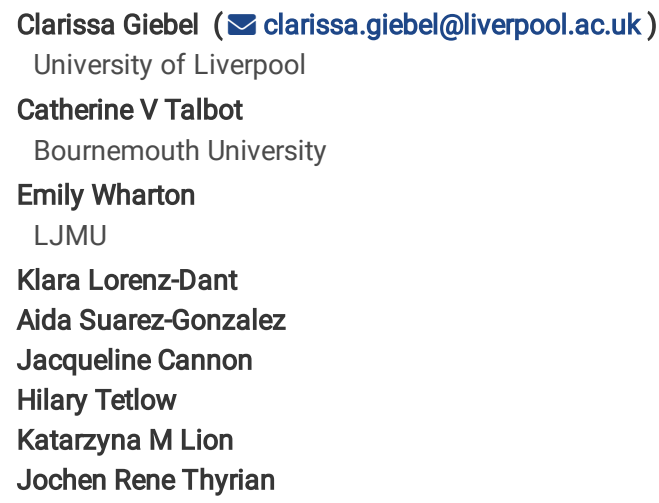




\section{Abstract \\ Background}

With a lack of existing comprehensive reviews, the aim of this mixed-methods systematic review was to synthesise the evidence on the early impacts of the pandemic on unpaid dementia carers across the globe.

\section{Methods}

This review was registered on PROSPERO [CDR42021248050]. PubMed, CINAHL, Embase, Scopus and Web of Science were searched from 2020 to July 2021. Studies were included if they reported on the different impacts of the pandemic on unpaid dementia carers aged 18+, with papers published in English, German, Polish, or Spanish. A number of research team members were involved in the selection of studies following PRISMA guidance.

\section{Results}

Thirty-six studies (43 papers) from 18 countries reported on the early impact of the pandemic on unpaid dementia carers. Impacts were noted on accessing care and support; carer burden; and well-being. Studies found that carers had limited access to care and support services, increased workload, enhanced feelings of isolation, and reduced wellbeing. All these negatives impacts were interlinked, with reductions in access to care and support increased carer's unpaid caring tasks, removing any opportunities for temporary respite, and thus increasing carer burden and reducing mental well-being in many.

\section{Conclusions}

The needs of unpaid dementia carers appear to have increased during the pandemic, without adequate support provided. Policy initiatives need to enable better mental health support and formal care provision for unpaid carers and their relatives with dementia, whilst future research needs to explore the longterm implications of carer needs in light of care home restrictions and care delivery.

\section{Introduction}

Of the estimated 55 million people living with dementia worldwide (WHO, 2021), many receive support from family members or friends, who are providing free and unpaid care to their relatives with dementia. Whilst providing a great deal of support for their relatives, unpaid carers are often overlooked in receiving support themselves (Clemmensen et al., 2021). Already before the COVID-19 pandemic, many unpaid carers experienced high levels of burden and poor mental well-being as a result (Sutcliffe et al., 2017). This increases as dementia advances, due to higher care needs of the person with dementia, and can often be a contributor for people with the condition to utilise more formal care including entering a care home (Kerpershoek et al., 2020).

People living with dementia have been particularly vulnerable and susceptible to the COVID-19 in this ongoing pandemic, due to their predominantly increased age (Banerjee et al., 2020) and frequent lack of understanding public health restrictions (Giebel et al., 2021a; Tuijt et al., 2021a). This has not only affected the person living with the condition, but also their support network which tries to keep them safe.

The COVID-19 pandemic has provided new care challenges and additional care needs for people with dementia, with social care and social support services primarily closed or operating at reduced levels (Giebel et al., 2021b). Early evidence seems to indicate that these reductions in external care support have led unpaid carers to take on additional care roles, on top of their previous caring roles (Rising et al., 2021; Sririam et al., 2021). Additional caring duties and hours without any reprise appear to be linked to poorer mental health and well-being in carers, with a growing body of evidence reporting on the wider exacerbated mental health needs of unpaid carers since the pandemic (Rainero et al., 2021; Vaitheswaran et al., 2021).

Despite a bourgeoning evidence base into the impacts of the pandemic on unpaid dementia carers, to date, it appears that no single systematic review has evaluated and synthesised the existing literature on the wider impacts of the pandemic on these unpaid carers. Instead, a rapid systematic review from early 2021 has looked at the psychological impact only, reporting negative effects of the pandemic on unpaid carers' mental health, including depression and anxiety (Hughes et al., 2021). The review failed to explore the wider impacts of the pandemic, however, on carers' access to care, which may be linked to reductions in health and well-being. Other systematic reviews on COVID-19 and dementia have solely focused on people with dementia and specific outcomes, such as cognition and mental health (Suarez-Gonzalez et al., 2021), or included non-primary research very early in the pandemic (Bacsu et al., 2021), providing little insights into the wider impacts on unpaid carers.

Therefore, the aim of this mixed-methods systematic review was to understand the impact of the COVID-19 pandemic on unpaid dementia carers, focusing on carers' health and well-being as well as health and social care access in the early stages of the pandemic. There is a continued need for understanding how the pandemic is impacting on unpaid carers, not just for dementia, but also for other conditions. COVID-19 is still affecting people's lives, so that knowledge from the early stages of the pandemic can help shaping care and support from Governments and care services to meet the needs of the unpaid workforce of some of the most vulnerable populations of our societies during the current pandemic as well as moving forward.

\section{Methods}

The protocol of this systematic review was registered on PROSPERO [ID: CRD42021248050]. Two unpaid carers (JC, HT) advised on the development of this review, were interpreting the findings jointly with other team members, read through drafts of the manuscript, and provided feedback. Due to the high number

Page $2 / 19$ 
of included studies, the results are presented in two parts: Part I focusing on people living with dementia and Part II focusing on unpaid carers. A full report of the methods for this review is presented in Part I (see Giebel et al., submitted). Only empirical studies were included in this review, both quantitative and

qualitative. Studies for Part I and II were included if they focused on people living with dementia aged 18+ and/or unpaid carers of people living with dementia aged 18+. Studies of people without a dementia diagnosis, formal or paid carers, or people aged 17 and younger were excluded. The data bases PubMed,

CINAHL, Embase, Scopus and Web of Science were searched from 2020 to July 2021, using the search term 'Covid-19' and search terms relating to dementia. Each title and abstract were screened by one reviewer (with the task divided amongst three reviewers due to citation size), and a $10 \%$ check was performed by a fourth reviewer as part of Stage 1. For Stage 2, each full-text paper was then screened by two reviewers each, with the task divided among four reviewers in total due to the large number of inclusions. Included studies were assessed using QualSyst (Kmet et al., 2004) by two researchers independently.

\section{Results}

\section{Overview of included studies and data selection}

159 full texts were read through for inclusion for the overarching review (Part I and II), with 36 studies reported in 43 papers specifically reporting on the impact of the pandemic on unpaid dementia carers. Studies were conducted across 18 countries, including Greece, Italy, Singapore, India, Poland, and the UK. The majority of studies $(n=25)$ were quantitative, including retrospective surveys enquiring about changes since the pandemic (Carpinelli-Mazzo et al., 2021; Pongan et al., 2021) and assessments of mental health and carer burden (i.e., Hwang et al., 2021; Losada et al., 2021). Qualitative studies (n=8) reported on remote interviews with unpaid carers about their experiences of providing care during the pandemic and their concerns (i.e., Rising et al., 2021; Sririram et al., 2021). Three mixed-methods studies reported on different impacts, including carer burden and access to care (Dassel et al., 2021; Rajagopalan et al., 2021; Savla et al., 2021). All studies focused on unpaid carers of community-residing people living with dementia, with Borg et al. (2021 focusing on both community and care homes.

[Figure 1; Tables 1-3] 
Table 1

Overview of included studies

\begin{tabular}{|c|c|c|c|c|c|c|c|c|}
\hline Authors & Country & $\begin{array}{l}\text { Population } \\
\text { (people with } \\
\text { dementia/carers/ } \\
\text { sample size) }\end{array}$ & Focus & Design & Measures & $\begin{array}{l}\text { Qual: themes } \\
\text { generated } \\
\text { Quant: outcomes }\end{array}$ & Setting & $\begin{array}{l}\text { Time } \\
\text { perio } \\
\text { data } \\
\text { coller }\end{array}$ \\
\hline $\begin{array}{l}\text { Alexopoulo et } \\
\text { al. }\end{array}$ & Greece & $\begin{array}{l}\text { Carers of people } \\
\text { with mild }(\mathrm{N}=13) \\
\text { and major }(\mathrm{N} \\
=54) \\
\text { neurocognitive } \\
\text { disorder (about } \\
\text { carers) }\end{array}$ & $\begin{array}{l}\text { Care } \\
\text { burden, } \\
\text { mental } \\
\text { health, } \\
\text { cognition }\end{array}$ & Quantitative & $\begin{array}{l}\text { Telephone survey was } \\
\text { based on items of the } \\
\text { neuropsychiatric inventory } \\
\text { questionnaire, the AD8 } \\
\text { Dementia Screening } \\
\text { Instrument, and the Bristol } \\
\text { Activities of Daily Living } \\
\text { Scale. Generalized Anxiety } \\
\text { Disorder 7-Item (GAD-7) } \\
\text { and the 22-item Impact of } \\
\text { Event Scale-revised (IES-R). }\end{array}$ & $\begin{array}{l}\text { Caregiver distress } \\
\text { severity during the } \\
\text { confinement } \\
\text { period was } \\
\text { influenced by } \\
\text { memory deficits, } \\
\text { neuropsychiatric } \\
\text { symptoms of } \\
\text { people with } \\
\text { dementia, } \\
\text { caregiver } \\
\text { hyperarousal and } \\
\text { avoidance } \\
\text { symptoms and } \\
\text { worries directly } \\
\text { linked to the } \\
\text { COVID-19 crisis. }\end{array}$ & Community & N/A \\
\hline $\begin{array}{l}\text { Altieri; } \\
\text { Santangelo }\end{array}$ & Italy & $\begin{array}{l}84 \text { carers of } \\
\text { people with } \\
\text { dementia (about } \\
\text { carers) }\end{array}$ & $\begin{array}{l}\text { Carer } \\
\text { burden, } \\
\text { mental } \\
\text { health }\end{array}$ & Quantitative & $\begin{array}{l}\text { Online survey, a } \\
\text { sociodemographic } \\
\text { questionnaire, the Italian } \\
\text { version of Hospital Anxiety } \\
\text { and } \\
\text { Depression Scale (HADS), } \\
\text { the Italian version of the } \\
\text { Resilience Scale for Adults } \\
\text { (RSA), the Italian version of } \\
\text { Caregiver Burden Inventory } \\
\text { (CBI). }\end{array}$ & $\begin{array}{l}\text { Produced } \\
\text { psychological } \\
\text { consequences in } \\
\text { carers of people } \\
\text { with dementia, } \\
\text { with an increase } \\
\text { of levels of } \\
\text { depression. High } \\
\text { resilience had a } \\
\text { negative effect on } \\
\text { anxiety levels. }\end{array}$ & Community & $\begin{array}{l}\text { April } \\
\text { and } ~ \\
\text { 3rd }\end{array}$ \\
\hline $\begin{array}{l}\text { Azevedo et } \\
\text { al. }\end{array}$ & $\begin{array}{l}\text { Argentina, } \\
\text { Brazil, } \\
\text { Chile }\end{array}$ & $\begin{array}{l}321 \text { family carers } \\
\text { of people with } \\
\text { dementia (about } \\
\text { people with } \\
\text { dementia and } \\
\text { carers) }\end{array}$ & $\begin{array}{l}\text { Care } \\
\text { Burden, } \\
\text { Mental } \\
\text { health, } \\
\text { Behaviour }\end{array}$ & $\begin{array}{l}\text { Quantitative } \\
\text { cross- } \\
\text { sectional } \\
\text { study }\end{array}$ & $\begin{array}{l}\text { Questionnaires were } \\
\text { applied via telephone - age, } \\
\text { sex, education level, } \\
\text { socioeconomic status } \\
\text { (through appropriate } \\
\text { instruments for each } \\
\text { Country), kinship, medical } \\
\text { diagnosis, and severity of } \\
\text { dementia. }\end{array}$ & $\begin{array}{l}\text { Individuals with } \\
\text { dementia felt } \\
\text { sadder and had } \\
\text { increased anxiety } \\
\text { symptoms. } \\
\text { Compulsive- } \\
\text { obsessive } \\
\text { behaviour, } \\
\text { hallucinations, } \\
\text { increased } \\
\text { forgetfulness, } \\
\text { altered appetite, } \\
\text { and increased } \\
\text { difficulty in } \\
\text { activities of daily } \\
\text { living were } \\
\text { reported more } \\
\text { frequently. Carers } \\
\text { reported feeling } \\
\text { more tired and } \\
\text { overwhelmed. }\end{array}$ & Community & $\begin{array}{l}\text { May: } \\
\text { July: }\end{array}$ \\
\hline Borelli et al. & Brazil & $\begin{array}{l}58 \text { people and } \\
\text { carers (about } \\
\text { people with } \\
\text { dementia and } \\
\text { carers) }\end{array}$ & $\begin{array}{l}\text { Care } \\
\text { burden, } \\
\text { mental } \\
\text { health, } \\
\text { cognition }\end{array}$ & $\begin{array}{l}\text { Quantitative } \\
\text { cross- } \\
\text { sectional } \\
\text { study }\end{array}$ & $\begin{array}{l}\text { The Neuropsychiatric } \\
\text { Inventory Questionnaire } \\
\text { (NPI-Q), Zarit Burden } \\
\text { Interview (ZBI), Beck } \\
\text { Depression (BDI) and } \\
\text { Anxiety (BAI) }\end{array}$ & $\begin{array}{l}\text { Cognitive decline } \\
\text { was shown, as } \\
\text { well as } \\
\text { behavioural } \\
\text { symptoms } \\
\text { especially } \\
\text { apathy/depression } \\
\text { and functional } \\
\text { decline. Increased } \\
\text { carers' } \\
\text { psychological } \\
\text { distress. }\end{array}$ & Community & $\begin{array}{l}\text { May: } \\
\text { to Ju } \\
2020\end{array}$ \\
\hline
\end{tabular}




\begin{tabular}{|c|c|c|c|c|c|c|c|c|}
\hline Authors & Country & $\begin{array}{l}\text { Population } \\
\text { (people with } \\
\text { dementia/carers/ } \\
\text { sample size) }\end{array}$ & Focus & Design & Measures & $\begin{array}{l}\text { Qual: themes } \\
\text { generated } \\
\text { Quant: outcomes }\end{array}$ & Setting & $\begin{array}{l}\text { Time } \\
\text { perio } \\
\text { data } \\
\text { colles }\end{array}$ \\
\hline Borg et al. & France & $\begin{array}{l}398 \text { carers } \\
\text { accompanying a } \\
\text { people with } \\
\text { dementia living } \\
\text { at home (HC) } \\
\text { and } 159 \\
\text { accompanying a } \\
\text { people with } \\
\text { dementia living } \\
\text { in a nursing } \\
\text { home (NHC) } \\
\text { (about people } \\
\text { with dementia } \\
\text { and carers) }\end{array}$ & $\begin{array}{l}\text { Care } \\
\text { burden, } \\
\text { Mental } \\
\text { health }\end{array}$ & $\begin{array}{l}\text { Quantitative } \\
\text { cross- } \\
\text { sectional } \\
\text { study }\end{array}$ & $\begin{array}{l}\text { Online survey - Anxiety was } \\
\text { assessed with the GAD-7 } \\
\text { scale, Depressive } \\
\text { symptoms were assessed } \\
\text { with the CES-D scale, } \\
\text { caregiver's burden was } \\
\text { assessed using the } \\
\text { validated short version of } \\
\text { the Zarit Burden Interview, } \\
\text { and level of self-rated } \\
\text { stress was assessed with a } \\
\text { visual analog scale } \\
\text { ranging from } 0 \text { (no stress } \\
\text { at all) to } \\
10 \text { (highest stress you can } \\
\text { imagine). }\end{array}$ & $\begin{array}{l}\text { Half of the carers } \\
\text { exhibited poor } \\
\text { mental health, } \\
\text { including } \\
\text { depression, } \\
\text { anxiety, or self- } \\
\text { reported stress. } \\
126 \text { PWD (34\%) } \\
\text { went to day care } \\
\text { facilities before } \\
\text { lockdown, this } \\
\text { type of } \\
\text { professional } \\
\text { support was } \\
\text { totally suspended } \\
\text { during the } \\
\text { lockdown. }\end{array}$ & $\begin{array}{l}\text { Community } \\
\text { and } \\
\text { nursing } \\
\text { home }\end{array}$ & $\begin{array}{l}17 \text { th } \\
\text { Marc } \\
11 \text { th } \\
2020\end{array}$ \\
\hline $\begin{array}{l}\text { Borges- } \\
\text { Machado et } \\
\text { al. }\end{array}$ & Portugal & $\begin{array}{l}36 \text { carers (about } \\
\text { people with } \\
\text { dementia and } \\
\text { carers) }\end{array}$ & $\begin{array}{l}\text { Care } \\
\text { burden, } \\
\text { mental } \\
\text { health }\end{array}$ & Quantitative & $\begin{array}{l}\text { Survey - Barthel Index (BI), } \\
\text { The NPI } 30 \text { was used to } \\
\text { analyse the BPSD, } \\
\text { CarerQol was used to } \\
\text { address Carer- } \\
\text { Qol-7D, and CarerQol-VAS. }\end{array}$ & $\begin{array}{l}\text { Increased } \\
\text { caregiving burden } \\
\text { and a decline in } \\
\text { their well-being. } \\
\text { People with } \\
\text { dementia } \\
\text { decreased their } \\
\text { volume of } \\
\text { physical activity, } \\
\text { and conversely } \\
\text { increased their } \\
\text { sitting time, } \\
\text { decreased well- } \\
\text { being. }\end{array}$ & Community & $\begin{array}{l}\text { Nove } \\
2019 \\
\text { June }\end{array}$ \\
\hline $\begin{array}{l}\text { Boutoleau- } \\
\text { Bretonnière et } \\
\text { al. ( } 2 \text { papers) }\end{array}$ & France & $\begin{array}{l}38 \text { carers (about } \\
\text { people with } \\
\text { Alzheimer's } \\
\text { disease and } \\
\text { carers) } \\
38 \text { behavioural } \\
\text { variant } \\
\text { frontotemporal } \\
\text { dementia (bvftd) } \\
\text { and } 38 \text { Alzheimer } \\
\text { disease (AD) } \\
\text { carers (about } \\
\text { carers) }\end{array}$ & $\begin{array}{l}\text { Cognition, } \\
\text { Mental } \\
\text { Health, care } \\
\text { burden } \\
\text { Behaviour, } \\
\text { Care } \\
\text { burden }\end{array}$ & Quantitative & $\begin{array}{l}\text { The Neuropsychiatric } \\
\text { Inventory-Questionnaire } \\
\text { Standardised surveys, } \\
\text { The Neuropsychiatric } \\
\text { Inventory (NPI) } \\
\text { Questionnaire }\end{array}$ & $\begin{array}{l}\text { Only } 10 \text { had } \\
\text { neuropsychiatric } \\
\text { changes. } \\
\text { Confinement } \\
\text { seems to impact } \\
\text { neuropsychiatric } \\
\text { symptomatology } \\
\text { in people with AD } \\
\text { with low baseline } \\
\text { cognitive function. } \\
\text { The duration of } \\
\text { confinement } \\
\text { significantly } \\
\text { correlated with } \\
\text { both the severity } \\
\text { of their } \\
\text { neuropsychiatric } \\
\text { symptoms, as well } \\
\text { as with the } \\
\text { distress } \\
\text { experienced by } \\
\text { their carers } \\
\text { Bvftd carers - } \\
\text { increased burden } \\
\text { regardless of } \\
\text { behavioural } \\
\text { changes. AD } \\
\text { carers -increased } \\
\text { burden related to } \\
\text { changes in people } \\
\text { with AD } \\
\text { neuropsychiatric } \\
\text { symptoms. }\end{array}$ & Community & $\begin{array}{l}26 \text { th } \\
\text { Marc } \\
9 \text { th N } \\
2020\end{array}$ \\
\hline $\begin{array}{l}\text { Carcavilla et } \\
\text { al. }\end{array}$ & Spain & $\begin{array}{l}106 \text { family carers } \\
\text { of people with } \\
\text { dementia (about } \\
\text { carers) }\end{array}$ & $\begin{array}{l}\text { Care } \\
\text { burden, } \\
\text { mental } \\
\text { health }\end{array}$ & $\begin{array}{l}\text { Quantitative } \\
\text { descriptive } \\
\text { cross- } \\
\text { sectional } \\
\text { study }\end{array}$ & $\begin{array}{l}\text { Online survey - Caregiver's } \\
\text { profile, main problems for } \\
\text { the caregiver and } \\
\text { consequences for both } \\
\text { people with dementia and } \\
\text { their carers during COVID, }\end{array}$ & $\begin{array}{l}\text { Family carers of } \\
\text { people with } \\
\text { dementia } \\
\text { experienced } \\
\text { psychological } \\
\text { problems, like } \\
\text { anxiety, mood, } \\
\text { sleep, or eating } \\
\text { disorders during } \\
\text { confinement. }\end{array}$ & Community & $\begin{array}{l}\text { May } \\
\text { June }\end{array}$ \\
\hline
\end{tabular}




\begin{tabular}{|c|c|c|c|c|c|c|c|c|}
\hline Authors & Country & $\begin{array}{l}\text { Population } \\
\text { (people with } \\
\text { dementia/carers/ } \\
\text { sample size) }\end{array}$ & Focus & Design & Measures & $\begin{array}{l}\text { Qual: themes } \\
\text { generated } \\
\text { Quant: outcomes }\end{array}$ & Setting & $\begin{array}{l}\text { Time } \\
\text { perio } \\
\text { data } \\
\text { colle }\end{array}$ \\
\hline $\begin{array}{l}\text { Carpinelli } \\
\text { Mazzi et al. }\end{array}$ & Italy & $\begin{array}{l}239 \text { carers of } \\
\text { people with } \\
\text { dementia (about } \\
\text { people with } \\
\text { dementia and } \\
\text { carers) }\end{array}$ & $\begin{array}{l}\text { Access to } \\
\text { care, } \\
\text { Mental } \\
\text { health, } \\
\text { Care } \\
\text { burden }\end{array}$ & Quantitative & $\begin{array}{l}\text { Telephone survey: Italian } \\
\text { versions of Zung's } \\
\text { depression and anxiety } \\
\text { assessment } \\
\text { Scales (ZDAAS), the } \\
\text { Perceived Stress Scale } \\
\text { (PSS), The ZBI (Zarit } \\
\text { Burden Interview) }\end{array}$ & $\begin{array}{l}\text { people with } \\
\text { dementia were } \\
\text { deprived of care } \\
\text { services and time } \\
\text { of isolation had a } \\
\text { significant } \\
\text { negative effect on } \\
\text { anxiety and } \\
\text { depression in } \\
\text { carers. }\end{array}$ & Community & N/A \\
\hline Cohen et al. & Argentina & $\begin{array}{l}119 \text { family carers } \\
\text { of persons with } \\
\text { AD or related } \\
\text { dementia } \\
\text { Living at home } \\
\text { (about people } \\
\text { with dementia } \\
\text { and carers) }\end{array}$ & $\begin{array}{l}\text { Behaviour, } \\
\text { mental } \\
\text { health, } \\
\text { health }\end{array}$ & Quantitative & $\begin{array}{l}\text { Questionnaire survey: } \\
\text { designed a visual analog } \\
\text { scale to study the burden } \\
\text { of care that } \\
\text { family members or paid } \\
\text { caregivers experienced } \\
\text { before and during the } \\
\text { epidemic, demographics of } \\
\text { family members, paid } \\
\text { caregivers, and dementia } \\
\text { subjects }\end{array}$ & $\begin{array}{l}\text { Increased anxiety } \\
\text { ( } 43 \% \text { of the } \\
\text { sample), insomnia } \\
\text { ( } 28 \% \text { of the } \\
\text { subjects), } \\
\text { depression }(29 \%) \text {, } \\
\text { worsening gait } \\
\text { disturbance ( } 41 \%) \text {, } \\
\text { and increase use } \\
\text { of psychotropics } \\
\text { to control } \\
\text { behavioural } \\
\text { symptoms. Social } \\
\text { isolation, lack of } \\
\text { outpatient } \\
\text { rehabilitation } \\
\text { services, and } \\
\text { increased stress of } \\
\text { family carers }\end{array}$ & Community & N/A \\
\hline Cohen et al. & Argentina & $\begin{array}{l}80 \text { family carers } \\
\text { of persons with } \\
\text { Alzheimer's } \\
\text { disease (about } \\
\text { people with } \\
\text { dementia and } \\
\text { carers) }\end{array}$ & $\begin{array}{l}\text { Mental } \\
\text { health, care } \\
\text { burden, } \\
\text { access to } \\
\text { care }\end{array}$ & Quantitative & $\begin{array}{l}\text { Questionnaire survey: } \\
\text { Items included } \\
\text { demographic } \\
\text { characteristics of } \\
\text { both subjects with } \\
\text { dementia and family } \\
\text { members } \\
\text { and problems of } \\
\text { management, } \\
\text { rehabilitation, and } \\
\text { care that subjects } \\
\text { experienced during the first } \\
4 \text { weeks of the coronavirus } \\
\text { quarantine in our setting, } \\
\text { anxiety, burnout and stress } \\
\text { that a family caregiver } \\
\text { experienced. }\end{array}$ & $\begin{array}{l}\text { Increased stress } \\
\text { caregiver, half of } \\
\text { the subjects with } \\
\text { dementia } \\
\text { experienced } \\
\text { increased anxiety } \\
\text { and that most } \\
\text { family members } \\
\text { discontinued all } \\
\text { sort of cognitive } \\
\text { and physical } \\
\text { therapies }\end{array}$ & Community & April \\
\hline Dassel et al. & USA & $\begin{array}{l}82 \text { carers (about } \\
\text { carers) }\end{array}$ & $\begin{array}{l}\text { Access to } \\
\text { care }\end{array}$ & Mixed & $\begin{array}{l}\text { Quantitative survey and } \\
\text { semi-structured qualitative } \\
\text { telephone interviews }\end{array}$ & $\begin{array}{l}\text { Some of family } \\
\text { carers revisited or } \\
\text { updated advance } \\
\text { directives of care } \\
\text { recipients and/or } \\
\text { had some type of } \\
\text { contingency plan } \\
\text { if they were to } \\
\text { become ill } 3 \\
\text { themes: } \\
\text { Interruption of } \\
\text { Care Plans, } \\
\text { focused on the } \\
\text { present, } \\
\text { navigating without } \\
\text { a compass }\end{array}$ & Community & $\begin{array}{l}\text { Surve } \\
\text { May } \\
\text { June } \\
2020 \\
\text { telepl } \\
\text { interv } \\
\text { July . } \\
\text { Sept€ } \\
2020\end{array}$ \\
\hline
\end{tabular}




\begin{tabular}{|c|c|c|c|c|c|c|c|c|}
\hline Authors & Country & $\begin{array}{l}\text { Population } \\
\text { (people with } \\
\text { dementia/carers/ } \\
\text { sample size) }\end{array}$ & Focus & Design & Measures & $\begin{array}{l}\text { Qual: themes } \\
\text { generated } \\
\text { Quant: outcomes }\end{array}$ & Setting & $\begin{array}{l}\text { Time } \\
\text { perio } \\
\text { data } \\
\text { coller }\end{array}$ \\
\hline Giebel et al. & UK & $\begin{array}{l}14 \text { unpaid carers, } \\
7 \text { people with } \\
\text { dementia (about } \\
\text { people with } \\
\text { dementia and } \\
\text { carers) }\end{array}$ & $\begin{array}{l}\text { Service } \\
\text { usage }\end{array}$ & $\begin{array}{l}\text { Qualitative } \\
\text { interviews }\end{array}$ & $\begin{array}{l}\text { Co-produced questions } \\
\text { about inequalities in } \\
\text { accessing post-diagnostic } \\
\text { care }\end{array}$ & $\begin{array}{l}4 \text { themes: } \\
\text { (1) Getting the ball } \\
\text { rolling: the process } \\
\text { of diagnosis; (2) } \\
\text { Balancing the } \\
\text { support needs of } \\
\text { people with } \\
\text { dementia and } \\
\text { carers; (3) Barriers } \\
\text { to accessing } \\
\text { support; and (4) } \\
\text { Facilitators to } \\
\text { accessing support. }\end{array}$ & Community & $\begin{array}{l}\text { Janu } \\
\text { Marc } \\
2020\end{array}$ \\
\hline $\begin{array}{l}\text { Giebel et al. } \\
\text { ( } 2 \text { papers) }\end{array}$ & UK & $\begin{array}{l}569 \text { participants, } \\
61 \text { people } \\
\text { with dementia, } \\
285 \text { unpaid } \\
\text { carers, and } 223 \\
\text { older adults } \\
\text { (about people } \\
\text { with dementia } \\
\text { and carers) }\end{array}$ & $\begin{array}{l}\text { Mental } \\
\text { wellbeing, } \\
\text { Service } \\
\text { usage }\end{array}$ & $\begin{array}{l}\text { Quantitative } \\
\text { 3-time point } \\
\text { survey }\end{array}$ & $\begin{array}{l}\text { Co-produced survey on } \\
\text { service usage, General } \\
\text { Health Questionnaire, } \\
\text { Short version of the } \\
\text { Warwick-Edinburgh Mental } \\
\text { Wellbeing Scale, Patient } \\
\text { Health Questionnaire }\end{array}$ & $\begin{array}{l}\text { Social support } \\
\text { service use } \\
\text { significantly } \\
\text { reduced since } \\
\text { pandemic; } \\
\text { Higher variations } \\
\text { in service usage } \\
\text { linked to increased } \\
\text { levels of anxiety in } \\
\text { people with } \\
\text { dementia, and } \\
\text { lower levels of } \\
\text { mental well-being } \\
\text { in unpaid carers. }\end{array}$ & Community & $\begin{array}{l}\text { April } \\
\text { Augu } \\
2020\end{array}$ \\
\hline $\begin{array}{l}\text { Giebel et al./ } \\
\text { Hanna et al. } \\
\text { (5 papers) }\end{array}$ & UK & $\begin{array}{l}50 \text { baseline } \\
\text { participants ( } 42 \\
\text { unpaid carers, } 8 \\
\text { people with } \\
\text { dementia), } 20 \\
\text { follow-up } \\
\text { interviews (16 } \\
\text { unpaid carers, } 4 \\
\text { people with } \\
\text { dementia) (about } \\
\text { people with } \\
\text { dementia and } \\
\text { carers) }\end{array}$ & $\begin{array}{l}\text { Service } \\
\text { usage, } \\
\text { Cognition, } \\
\text { Everyday } \\
\text { functioning, } \\
\text { Mental } \\
\text { wellbeing }\end{array}$ & $\begin{array}{l}\text { Qualitative } \\
\text { interviews }\end{array}$ & $\begin{array}{l}\text { Co-produced questions } \\
\text { about service usage before } \\
\text { and since the pandemic }\end{array}$ & $\begin{array}{l}\text { People with } \\
\text { dementia were } \\
\text { reported to } \\
\text { deteriorate faster, } \\
\text { struggled } \\
\text { accessing social } \\
\text { support services, } \\
\text { and experienced } \\
\text { difficulties } \\
\text { deciding about } \\
\text { whether to receive } \\
\text { paid home care. } \\
\text { Inequalities in } \\
\text { accessing care } \\
\text { noted. Mental } \\
\text { health issues } \\
\text { noted for people } \\
\text { with dementia and } \\
\text { unpaid carers. }\end{array}$ & Community & $\begin{array}{l}\text { April } \\
\text { July : }\end{array}$ \\
\hline $\begin{array}{l}\text { Helvaci } \\
\text { Yilmaz et al. }\end{array}$ & Turkey & $\begin{array}{l}54 \text { individuals } \\
\text { diagnosed with } \\
\text { AD and carers } \\
\text { (about people } \\
\text { with dementia } \\
\text { and carers) }\end{array}$ & $\begin{array}{l}\text { Carer } \\
\text { burden, } \\
\text { cognition, } \\
\text { mental } \\
\text { health }\end{array}$ & Quantitative & $\begin{array}{l}\text { Survey prepared by the } \\
\text { neurology department of } \\
\text { Medipol University Istanbu }\end{array}$ & $\begin{array}{l}\text { AD worsening } \\
\text { symptom was } \\
\text { forgetfulness and } \\
\text { agitation. The } \\
\text { carers thought } \\
\text { that something } \\
\text { terrible would } \\
\text { happen to the } \\
\text { patient and felt } \\
\text { they could not find } \\
\text { time for } \\
\text { themselves. Drug } \\
\text { rejection increased } \\
\text { the burden twofold }\end{array}$ & Community & $\begin{array}{l}\text { April } \\
\text { May } \\
2020\end{array}$ \\
\hline Hwang et al. & USA & $\begin{array}{l}34 \text { carers (about } \\
\text { carers) }\end{array}$ & $\begin{array}{l}\text { Mental } \\
\text { health, care } \\
\text { burden }\end{array}$ & Quantitative & $\begin{array}{l}\text { Questions were developed } \\
\text { by the authors based on } \\
\text { the previous literature, } \\
\text { generalized Anxiety } \\
\text { Disorder-7, Center for } \\
\text { Epidemiologic Studies } \\
\\
\text { Depression Scale, Clinical } \\
\text { Dementia Rating (CDR), } \\
\text { Barthel Activities of Daily } \\
\text { Living Index, } \\
\text { Neuropsychiatric Inventory } \\
\text { (NPI). }\end{array}$ & $\begin{array}{l}\text { Worrying about } \\
\text { spreading covid- } \\
19 \text { to the people } \\
\text { with dementia, } \\
\text { taken on } \\
\text { additional } \\
\text { caregiving duties } \\
\text { for others in their } \\
\text { family since covid- } \\
19 \text {, and reported } \\
\text { one or more } \\
\text { anxiety symptoms }\end{array}$ & Community & $\begin{array}{l}\text { June } \\
2020 \\
\text { Augu } \\
28 \text { th, } \\
2020\end{array}$ \\
\hline
\end{tabular}




\begin{tabular}{|c|c|c|c|c|c|c|c|c|}
\hline Authors & Country & $\begin{array}{l}\text { Population } \\
\text { (people with } \\
\text { dementia/carers/ } \\
\text { sample size) }\end{array}$ & Focus & Design & Measures & $\begin{array}{l}\text { Qual: themes } \\
\text { generated } \\
\text { Quant: outcomes }\end{array}$ & Setting & $\begin{array}{l}\text { Time } \\
\text { perio } \\
\text { data } \\
\text { colle }\end{array}$ \\
\hline Kostyal et al. & Hungary & $\begin{array}{l}370 \text { family carers } \\
\text { of people with } \\
\text { dementia (about } \\
\text { carers) }\end{array}$ & $\begin{array}{l}\text { Access to } \\
\text { care, care } \\
\text { burden, } \\
\text { mental } \\
\text { health }\end{array}$ & Quantitative & $\begin{array}{l}\text { Online survey - socio- } \\
\text { demographic background, } \\
\text { caregiver-care recipient } \\
\text { relationship, caregiver's } \\
\text { responsibilities: caregiver's } \\
\text { self-reported physical, } \\
\text { general and mental health } \\
\text { status, difficulties posed by } \\
\text { the pandemic }\end{array}$ & $\begin{array}{l}\text { A deterioration in } \\
\text { their financial } \\
\text { status. A decline in } \\
\text { general and } \\
\text { mental health, } \\
\text { family carers' } \\
\text { burden increased }\end{array}$ & Community & $\begin{array}{l}\text { May- } \\
2020\end{array}$ \\
\hline Losada et al. & Spain & $\begin{array}{l}88 \text { family carers } \\
\text { (about family } \\
\text { caregiver) }\end{array}$ & $\begin{array}{l}\text { Care } \\
\text { burden, } \\
\text { Cognition, } \\
\text { Mental } \\
\text { health }\end{array}$ & Quantitative & $\begin{array}{l}\text { Telephone survey - } \\
\text { Spanish version of GDS } \\
\text { and Barthel Index- } \\
\text { Behavioural and } \\
\text { psychological symptoms, } \\
\text { Carers' depressive and } \\
\text { anxiety symptomatology, } \\
\text { carers emotions, family } \\
\text { and economy during } \\
\text { COVID }\end{array}$ & $\begin{array}{l}\text { Increased negative } \\
\text { emotions, } \\
\text { conflicts, and } \\
\text { thoughts on giving } \\
\text { up on caregiving. } \\
\text { Some carers had } \\
\text { increased positive } \\
\text { emotions. }\end{array}$ & Community & $\begin{array}{l}\text { June } \\
- \text { Mic } \\
\text { of Mi } \\
2021\end{array}$ \\
\hline $\mathrm{Ng}$ et al. & Singapore & $\begin{array}{l}50 \text { people with } \\
\text { Mild FTD and } 50 \\
\text { people with mild } \\
\text { AD dementia } \\
\text { (about people } \\
\text { with dementia } \\
\text { and carers) }\end{array}$ & $\begin{array}{l}\text { Access to } \\
\text { care, } \\
\text { behaviour }\end{array}$ & Quantitative & $\begin{array}{l}\text { Phone based } \\
\text { Survey - to identify major } \\
\text { themes of particular } \\
\text { concern to patient-carers } \\
\text { dyads of FTD. }\end{array}$ & $\begin{array}{l}\text { Preliminary } \\
\text { findings } \\
\text { Demonstrate that } \\
\text { people with FTD } \\
\text { have significant } \\
\text { worsening in } \\
\text { behaviour and } \\
\text { social cognition, } \\
\text { as well as suffer } \\
\text { greater negative } \\
\text { consequences } \\
\text { from disruption to } \\
\text { healthcare } \\
\text { services compared } \\
\text { to people with AD. }\end{array}$ & Community & N/A \\
\hline Panerai et al. & Italy & $\begin{array}{l}\text { 128carers of } \\
\text { people with } \\
\text { dementia (about } \\
\text { people with } \\
\text { dementia and } \\
\text { carers) }\end{array}$ & $\begin{array}{l}\text { Care } \\
\text { burden, } \\
\text { cognition, } \\
\text { mental } \\
\text { health }\end{array}$ & Quantitative & $\begin{array}{l}\text { The Neuropsychiatric } \\
\text { Inventory Questionnaire } \\
\text { (NPI-Q) and the Caregiver } \\
\text { Burden Inventory (CBI) }\end{array}$ & $\begin{array}{l}\text { Increased risk for } \\
\text { burning out of } \\
\text { carers, } \\
\text { Neuropsychiatric } \\
\text { symptoms in } \\
\text { people with } \\
\text { dementia } \\
\text { significantly } \\
\text { increased as well. }\end{array}$ & Community & $\begin{array}{l}\text { April } \\
\text { and } ~ \\
\text { 16th }\end{array}$ \\
\hline Pongan et al. & France & $\begin{array}{l}\text { Carers of people } \\
\text { with dementia } \\
\text { (about people } \\
\text { with dementia } \\
\text { and carers) }\end{array}$ & $\begin{array}{l}\text { Care } \\
\text { burden, } \\
\text { behaviour, } \\
\text { Mental } \\
\text { health }\end{array}$ & Quantitative & $\begin{array}{l}\text { Cross-sectional online } \\
\text { survey, The Checklist for } \\
\text { Reporting Results of } \\
\text { Internet E-Surveys } \\
\text { (CHERRIES), the GAD-7 } \\
\text { scale (The General Anxiety } \\
\text { Disorder-7), The CES-D } \\
\text { scale (Center for } \\
\text { Epidemiologic Studies- } \\
\text { Depression) }\end{array}$ & $\begin{array}{l}\text { Impact on } \\
\text { behavioural } \\
\text { disorders in people } \\
\text { with dementia and } \\
\text { these disorders are } \\
\text { associated with } \\
\text { poorer mental } \\
\text { health of carers. }\end{array}$ & Community & $\begin{array}{l}\text { 15th } \\
-15 t \\
\text { June }\end{array}$ \\
\hline Rainero et al. & Italy & $\begin{array}{l}97 \text { dementia } \\
\text { centers, } 4913 \\
\text { people with } \\
\text { dementia (about } \\
\text { people with } \\
\text { dementia and } \\
\text { carers) }\end{array}$ & $\begin{array}{l}\text { Care } \\
\text { burden, } \\
\text { mental } \\
\text { health }\end{array}$ & Quantitative & $\begin{array}{l}\text { Survey, Clinical Dementia } \\
\text { Rating (CDR) }\end{array}$ & $\begin{array}{l}\text { Carers reported a } \\
\text { high increase in } \\
\text { anxiety, } \\
\text { depression, and } \\
\text { distress. Acute } \\
\text { worsening of } \\
\text { clinical symptoms } \\
\text { in people with } \\
\text { dementia }\end{array}$ & Community & April \\
\hline $\begin{array}{l}\text { Rajagopalan } \\
\text { et al. }\end{array}$ & India & $\begin{array}{l}104 \text { dementia } \\
\text { and their carers } \\
\text { (about people } \\
\text { with dementia } \\
\text { and carers) }\end{array}$ & $\begin{array}{l}\text { Care } \\
\text { burden, } \\
\text { behaviour, } \\
\text { mental } \\
\text { health, } \\
\text { access to } \\
\text { care }\end{array}$ & $\begin{array}{l}\text { Mixed } \\
\text { method }\end{array}$ & $\begin{array}{l}\text { Validated instruments and } \\
\text { a semi-structured interview } \\
\text { guide }\end{array}$ & $\begin{array}{l}\text { Worsening of } \\
\text { behaviour, } \\
\text { problems in } \\
\text { accessing care, } \\
\text { disruptions in } \\
\text { functional } \\
\text { activities and } \\
\text { struggles in } \\
\text { enforcing infection } \\
\text { prevention } \\
\text { contributing to } \\
\text { caregiver distress. }\end{array}$ & Community & $\begin{array}{l}15 \mathrm{M} \\
\text { and } 2 \\
\text { June } \\
2020\end{array}$ \\
\hline
\end{tabular}




\begin{tabular}{|c|c|c|c|c|c|c|c|c|}
\hline Authors & Country & $\begin{array}{l}\text { Population } \\
\text { (people with } \\
\text { dementia/carers/ } \\
\text { sample size) }\end{array}$ & Focus & Design & Measures & $\begin{array}{l}\text { Qual: themes } \\
\text { generated } \\
\text { Quant: outcomes }\end{array}$ & Setting & $\begin{array}{l}\text { Time } \\
\text { perio } \\
\text { data } \\
\text { coller }\end{array}$ \\
\hline Rising et al. & USA & $\begin{array}{l}25 \text { people with } \\
\text { dementia and } \\
\text { Caregiver (about } \\
\text { people with } \\
\text { dementia and } \\
\text { carers) }\end{array}$ & $\begin{array}{l}\text { Care } \\
\text { burden, } \\
\text { cognition, } \\
\text { mental } \\
\text { health }\end{array}$ & Qualitative & Semi-structured interviews & $\begin{array}{l}\text { Four themes } \\
\text { emerging: } \\
\text { disruption of } \\
\text { socialization, fear } \\
\text { and risk } \\
\text { mitigation, coping } \\
\text { strategies, and } \\
\text { caregiver burden }\end{array}$ & Community & $\begin{array}{l}\text { Augu } \\
\text { Nove } \\
2020\end{array}$ \\
\hline $\begin{array}{l}\text { Rusowicz et } \\
\text { al. }\end{array}$ & Poland & $\begin{array}{l}85 \text { carers (about } \\
\text { caregiver) }\end{array}$ & $\begin{array}{l}\text { Care } \\
\text { burden, } \\
\text { mental } \\
\text { health }\end{array}$ & Quantitative & $\begin{array}{l}\text { Questionnaire; 10-item } \\
\text { Perceived Stress Scale } \\
\text { (PSS-10) }\end{array}$ & $\begin{array}{l}\text { High levels of } \\
\text { stress, PSS-10 } \\
\text { correlated with the } \\
\text { deterioration of } \\
\text { illness during } \\
\text { Covid-19, changes } \\
\text { in daily } \\
\text { functioning, and } \\
\text { concerns about } \\
\text { both the health of } \\
\text { the charge and } \\
\text { caregiver }\end{array}$ & Community & $\mathrm{N} / \mathrm{A}$ \\
\hline Savla et al. & USA & $\begin{array}{l}53 \text { family carers } \\
\text { of persons with } \\
\text { dementia (about } \\
\text { caregiver) }\end{array}$ & $\begin{array}{l}\text { Cognition, } \\
\text { Care } \\
\text { burden, } \\
\text { access to } \\
\text { care }\end{array}$ & Mixed & $\begin{array}{l}\text { Structured survey and } \\
\text { open-ended questions - } \\
\text { people with dementia's } \\
\text { living arrangement and } \\
\text { cgs' relationship with } \\
\text { people with dementia, age, } \\
\text { perception of income } \\
\text { adequacy, and } \\
\text { race/ethnicity, people with } \\
\text { dementia's memory-related } \\
\text { behaviour problems, cgs } \\
\text { rated three items } \\
\text { exhausted, more things to } \\
\text { do than can handle, didn't } \\
\text { have time for self) on a 4- } \\
\text { point Likert scale from } 0 \\
\text { (not at all) to } 3 \text { (extremely) }\end{array}$ & $\begin{array}{l}\text { Carers who were } \\
\text { more concerned } \\
\text { about the COVID- } \\
19 \text { pandemic were } \\
\text { at greater odds of } \\
\text { experiencing high } \\
\text { role overload than } \\
\text { those who } \\
\text { recognized } \\
\text { positive aspects of } \\
\text { the pandemic. } \\
\text { Home care } \\
\text { services had } \\
\text { reduced. }\end{array}$ & Community & $\begin{array}{l}\text { April } \\
\text { and } \uparrow\end{array}$ \\
\hline Sriram et al. & UK & $\begin{array}{l}23 \text { carers (about } \\
\text { carers) }\end{array}$ & $\begin{array}{l}\text { Care } \\
\text { burden, } \\
\text { access to } \\
\text { care, } \\
\text { mental } \\
\text { health }\end{array}$ & Qualitative & Semi-structured Interview & $\begin{array}{l}\text { Three main } \\
\text { themes-Changes } \\
\text { to daily life, } \\
\text { impact on carer } \\
\text { health and } \\
\text { wellbeing and } \\
\text { reduced support } \\
\text { from health and } \\
\text { social support } \\
\text { networks. }\end{array}$ & Community & $\mathrm{N} / \mathrm{A}$ \\
\hline Tam et al. & Canada & $\begin{array}{l}498 \text { participants } \\
\text { carers and } \\
\text { people with } \\
\text { dementia (about } \\
\text { people with } \\
\text { dementia and } \\
\text { carers) }\end{array}$ & $\begin{array}{l}\text { Carer } \\
\text { burden, } \\
\text { health } \\
\text { services, } \\
\text { mental } \\
\text { health }\end{array}$ & Quantitative & $\begin{array}{l}\text { Survey - themes: 1) } \\
\text { information and resource } \\
\text { needs, 2) } \\
\text { Caring for someone living } \\
\text { with dementia during the } \\
\text { COVID-19 pandemic } \\
\text { (specific to care partner } \\
\text { surveys), } \\
\text { 3) mental health and well- } \\
\text { being needs, 4) the use of } \\
\text { technology for social } \\
\text { connection during the } \\
\text { pandemic. }\end{array}$ & $\begin{array}{l}\text { Reported several } \\
\text { serious concerns, } \\
\text { inability to visit the } \\
\text { person that they } \\
\text { care for in long- } \\
\text { term or palliative } \\
\text { care. Reported that } \\
\text { the pandemic } \\
\text { increased their } \\
\text { levels of stress } \\
\text { overall and that } \\
\text { they felt lonelier } \\
\text { and more isolated } \\
\text { than they did } \\
\text { before the } \\
\text { pandemic. }\end{array}$ & Community & $\begin{array}{l}\text { June } \\
2020 \\
\text { Augu } \\
2020\end{array}$ \\
\hline $\begin{array}{l}\text { Tsapanou et } \\
\text { al. }\end{array}$ & Greece & $\begin{array}{l}339 \text { carers } \\
\text { (about carers } \\
\text { and people with } \\
\text { dementia) }\end{array}$ & $\begin{array}{l}\text { Carer } \\
\text { burden }\end{array}$ & $\begin{array}{l}\text { Quantitative, } \\
\text { Exploratory } \\
\text { sequential } \\
\text { mixed- } \\
\text { methods } \\
\text { design - } \\
\text { Quantitative }\end{array}$ & $\begin{array}{l}\text { Online questionnaire was } \\
\text { created regarding both } \\
\text { changes of the patient they } \\
\text { take care of, and changes } \\
\text { to their own burden }\end{array}$ & $\begin{array}{l}\text { Significant decline, } \\
\text { both in an overall } \\
\text { aspect of the } \\
\text { people with } \\
\text { dementia, and in } \\
\text { specific domains } \\
\text { (mostly } \\
\text { communication } \\
\text { and mood). carers } \\
\text {-significantly } \\
\text { increased physical } \\
\text { and psychological } \\
\text { burden }\end{array}$ & Community & $\begin{array}{l}\text { Nove } \\
2020 \\
\text { April }\end{array}$ \\
\hline
\end{tabular}




\begin{tabular}{|c|c|c|c|c|c|c|c|c|}
\hline Authors & Country & $\begin{array}{l}\text { Population } \\
\text { (people with } \\
\text { dementia/carers/ } \\
\text { sample size) }\end{array}$ & Focus & Design & Measures & $\begin{array}{l}\text { Qual: themes } \\
\text { generated } \\
\text { Quant: outcomes }\end{array}$ & Setting & $\begin{array}{l}\text { Time } \\
\text { perio } \\
\text { data } \\
\text { colle }\end{array}$ \\
\hline $\begin{array}{l}\text { Tuijt et al. ( } 2 \\
\text { papers) }\end{array}$ & UK & $\begin{array}{l}30 \text { people living } \\
\text { with dementia, } \\
31 \\
\text { Family carer } \\
\text { (about people } \\
\text { with dementia } \\
\text { and carers) }\end{array}$ & $\begin{array}{l}\text { Cognition, } \\
\text { care burden } \\
\text { Access to } \\
\text { care }\end{array}$ & Qualitative & $\begin{array}{l}\text { Interviews were semi } \\
\text { structured } \\
\text { Semi-structured interviews }\end{array}$ & $\begin{array}{l}\text { 1) awareness of } \\
\text { restrictions, 2) } \\
\text { restructuring } \\
\text { caring } \\
\text { relationships to } \\
\text { manage covid-19 } \\
\text { risk, 3) protective } \\
\text { factors, 4) the } \\
\text { psychological and } \\
\text { cognitive impact } \\
\text { of restrictions, and } \\
\text { 5) the importance } \\
\text { of social } \\
\text { engagement. } \\
\text { Three main } \\
\text { themes were } \\
\text { derived relating to: } \\
\text { proactive care at } \\
\text { the onset of covid- } \\
\text { 19 restrictions; } \\
\text { avoidance of } \\
\text { healthcare } \\
\text { settings and } \\
\text { services; and } \\
\text { difficulties with } \\
\text { remote healthcare } \\
\text { encounters. }\end{array}$ & Community & $\begin{array}{l}\text { May } \\
\text { Augu } \\
2020\end{array}$ \\
\hline Tulloch et al. & Australia & $\begin{array}{l}26 \text { carers of } \\
\text { people with } \\
\text { dementia (about } \\
\text { carers) }\end{array}$ & $\begin{array}{l}\text { Access to } \\
\text { care, } \\
\text { positive } \\
\text { caregiver } \\
\text { experiences }\end{array}$ & Qualitative & Semi-structured interviews & $\begin{array}{l}\text { Pre-pandemic } \\
\text { care: (1) focusing } \\
\text { on the people with } \\
\text { dementia and (2) } \\
\text { working together } \\
\text { during-pandemic } \\
\text { care: (1) } \\
\text { respecting } \\
\text { personhood; (2) } \\
\text { connecting with } \\
\text { virtues and values; } \\
\text { (3) improving } \\
\text { relationships; (4) } \\
\text { seeking and } \\
\text { receiving support; } \\
\text { (5) prioritizing self- } \\
\text { care; (6) being } \\
\text { protective and } \\
\text { proactive; (7) } \\
\text { making practical } \\
\text { changes. Moving } \\
\text { forward from } \\
\text { COVID-19: (1) } \\
\text { strengthening } \\
\text { commitment to } \\
\text { the people with } \\
\text { dementia (2) } \\
\text { looking after my } \\
\text { own needs, and } \\
\text { (3) considering } \\
\text { practical } \\
\text { requirements. }\end{array}$ & Community & $\begin{array}{l}\text { June } \\
\text { and } \\
\text { Augu } \\
2020\end{array}$ \\
\hline $\begin{array}{l}\text { Vaitheswaran } \\
\text { et al. }\end{array}$ & India & $\begin{array}{l}31 \text { carers (about } \\
\text { carers) }\end{array}$ & $\begin{array}{l}\text { Care } \\
\text { burden, } \\
\text { carers need }\end{array}$ & Qualitative & Semi structured interviews & $\begin{array}{l}\text { Themes: unique to } \\
\text { the carers that } \\
\text { directly related to } \\
\text { their caregiving } \\
\text { role, while the } \\
\text { second set did not } \\
\text { relate directly to } \\
\text { their caregiving } \\
\text { role, needs, } \\
\text { provision of } \\
\text { support to meet } \\
\text { needs }\end{array}$ & Community & $\begin{array}{l}\text { 1st } \\
\text { Septt } \\
2019 \\
29 \text { th } \\
\text { Febrt } \\
2020\end{array}$ \\
\hline
\end{tabular}




\begin{tabular}{|c|c|c|c|c|c|c|c|c|}
\hline Authors & Country & $\begin{array}{l}\text { Population } \\
\text { (people with } \\
\text { dementia/carers/ } \\
\text { sample size) }\end{array}$ & Focus & Design & Measures & $\begin{array}{l}\text { Qual: themes } \\
\text { generated } \\
\text { Quant: outcomes }\end{array}$ & Setting & $\begin{array}{l}\text { Time } \\
\text { perio } \\
\text { data } \\
\text { coller }\end{array}$ \\
\hline Werner et al. & Israel & $\begin{array}{l}73 \text { Israeli family } \\
\text { carers of } \\
\text { Persons with } \\
\text { dementia (about } \\
\text { carers) }\end{array}$ & $\begin{array}{l}\text { Behaviour, } \\
\text { access to } \\
\text { care }\end{array}$ & $\begin{array}{l}\text { Quantitative } \\
\text { cross- } \\
\text { sectional } \\
\text { study }\end{array}$ & $\begin{array}{l}\text { Online survey - Problematic } \\
\text { Behaviour Scale, } \\
\text { loneliness, cognitive } \\
\text { function, fear }\end{array}$ & $\begin{array}{l}\text { Feelings of burden } \\
\text { Were associated } \\
\text { with carers' } \\
\text { forgone care and } \\
\text { feelings of } \\
\text { loneliness and } \\
\text { perceptions of the } \\
\text { care-receiver's } \\
\text { cognitive } \\
\text { functioning were } \\
\text { associated with } \\
\text { care-receivers' } \\
\text { forgone care. }\end{array}$ & Community & $\begin{array}{l}25 \mathrm{~J} \\
2020 \\
\\
\text { And } \\
\text { close } \\
25 \mathrm{JL} \\
2020\end{array}$ \\
\hline West et al. & UK & $\begin{array}{l}15 \text { participants } \\
\text { (people with } \\
\text { dementia and } \\
\text { Carers) }\end{array}$ & $\begin{array}{l}\text { Health, } \\
\text { access to } \\
\text { care, } \\
\text { Mental } \\
\text { health, care } \\
\text { burden }\end{array}$ & Qualitative & $\begin{array}{l}\text { Semi structured qualitative } \\
\text { Interviews. }\end{array}$ & $\begin{array}{l}8 \text { key themes, with } \\
\text { subthemes: Fear } \\
\text { and anxiety, food } \\
\text { and eating } \\
\text { (encompassing } \\
\text { food } \\
\text { Shopping and } \\
\text { eating patterns), } \\
\text { isolation and } \\
\text { identity, } \\
\text { Community and } \\
\text { social } \\
\text { relationships, } \\
\text { adapting to covid- } \\
\text { 19, social isolation } \\
\text { and support } \\
\text { structures, and } \\
\text { medical } \\
\text { interactions. }\end{array}$ & Community & N/A \\
\hline Zucca et al. & Italy & $\begin{array}{l}87 \text { dementia } \\
\text { centres, } 4710 \\
\text { caregiver - } \\
\text { people with } \\
\text { dementia pairs } \\
\text { (about carers) }\end{array}$ & $\begin{array}{l}\text { Care } \\
\text { burden, } \\
\text { Mental } \\
\text { Health }\end{array}$ & Quantitative & $\begin{array}{l}\text { Survey: six } \\
\text { Questions tapping the } \\
\text { following stress } \\
\text { symptoms: } \\
\text { (1) depression, (2) anxiety, } \\
(3) \text { anguish, (4) irritability, } \\
\text { (5) } \\
\text { overwhelmed/helplessness } \\
\text { (OH), and (6) } \\
\text { isolation/abandonment } \\
\text { (IA). }\end{array}$ & $\begin{array}{l}\text { Increased stress, } \\
\text { anxiety, concerned } \\
\text { for people with } \\
\text { dementia' health, } \\
\text { sense of isolation } \\
\text { and } \\
\text { abandonment. }\end{array}$ & Community & $\begin{array}{l}\text { April } \\
14 \text { to } \\
24,21\end{array}$ \\
\hline
\end{tabular}


Table 2

Quality assessment ratings for quantitative studies

\begin{tabular}{|c|c|}
\hline Research Paper & Overall Score \\
\hline Alexopoulo et al. & 1.00 \\
\hline Altieri; Santangelo & 0.89 \\
\hline Azevedo et al. & 0.94 \\
\hline Borelli et al. & 1.00 \\
\hline Borg et al. & 0.94 \\
\hline Borges-Machado et al. & 0.94 \\
\hline Boutoleau-Bretonnière et al. & 1.00 \\
\hline Carcavilla et al. & 0.67 \\
\hline Carpinelli Mazzi et al. & 0.78 \\
\hline Cohen et al. & 1.00 \\
\hline Cohen et al. & 0.89 \\
\hline Dassel et al. & 0.71 \\
\hline El haj et al. & 0.83 \\
\hline El haj et al. & 0.83 \\
\hline Giebel et al. (2 papers) & 1.00 \\
\hline Helvaci yilmaz et al. & 0.94 \\
\hline Hwang et al. & 1.00 \\
\hline Kostyal et al. & 0.89 \\
\hline Losada et al. & 1.00 \\
\hline $\mathrm{Ng}$ et al. & 0.78 \\
\hline Panerai et al. & 1.00 \\
\hline Pongan et al. & 0.94 \\
\hline Rainero et al. & 1.00 \\
\hline Rajagopalan et al. & 0.94 \\
\hline Rusowicz et al. & 0.94 \\
\hline Savla et al. & 0.82 \\
\hline Tam et al. & 0.89 \\
\hline Tsapanou et al. & 0.89 \\
\hline Werner et al. & 0.94 \\
\hline Zucca et al. & 0.94 \\
\hline
\end{tabular}


Table 3

Quality assessment ratings for qualitative studies

\begin{tabular}{|ll|}
\hline Research Paper & Overall Score \\
\hline Dassel et al. & 0.70 \\
\hline Giebel et al. & 0.95 \\
\hline Giebel et al./ Hanna et al. (5 papers) & 0.90 \\
\hline Rajagopalan et al. & 0.85 \\
\hline Rising et al. & 0.65 \\
\hline Savla et al. & 0.75 \\
\hline Sriram et al. & 1.00 \\
\hline Talbot; Briggs & 0.90 \\
\hline Tuijt et al. (2 papers) & 0.90 \\
\hline Tulloch et al. & 0.85 \\
\hline Vaitheswaran et al. & 0.65 \\
\hline West et al. & 0.90 \\
\hline
\end{tabular}

\section{Impact on access to care and support}

The impact of the pandemic on access to health or social care was reported in 14 studies, all of which identified reductions in access for unpaid carers, leaving many people without access to vital support. The availability of support services also appeared to be location-dependent, with level of support services varying greatly between areas, based on UK reports (Giebel, Hanna et al., 2021; West et al., 2021). Carers reported reduced access to all areas of support services, including local day centres, memory cafes, support groups and respite care worldwide (Carpinelle-Mazi et al., 2021a; Cohen et al., 2021a; Rajagopalan et al., 2021). Most of those support the person with dementia directly and the unpaid carer indirectly, whilst peer support groups can support either (e.g., Giebel et al., 2020; Sriram et al., 2021). Following up with carers at two subsequent time points, Giebel et al. (2021b) identified a small upward trend in access to social support services again in the months following the first nationwide lockdown in the UK, with access to paid carers being the least affected by the pandemic. Paid home care may have been the least impacted service because of the difficult decisions carers needed to make during the pandemic, whereby some carers suspended paid care visits due to fears of transmitting COVID-19, while others felt they could not cope without paid care and were fearful of reobtaining it post-pandemic (Giebel et al., 2020). Similar findings in access to care during the pandemic were reported in other studies (e.g., Cohen et al., 2020a; Dassel et al., 2021; Rajagopalan et al., 2021; Sriram et al., 2021; Tuijt et al., 2021b). However, there were some reports of local area agencies on ageing checking in on carers more frequently than before COVID-19 and nutrition services delivering extra meals (e.g., Savla et al., 2020).

Difficulties with accessing care homes due to the increased care needs of the care-recipient was reported in one study (Giebel et al., 2021c). In this UK study, carers expressed that finding care home places was challenging due to inflated fees and care home closures, with some carers opting for a care home place far away.

Access to healthcare services was explored in some studies. In Israel, Werner et al. (2021) found that $50 \%$ of the carers who needed to see a GP or specialist had forgone at least one of these services. In Argentina, carers reported to have discontinued all sorts of cognitive and physical therapies (Cohen et al., 2020a). Similarly, in an interview study by Tuijt et al. (2021b), carers reported avoiding healthcare settings and services due to the risk of coronavirus transmission and fears of overburdening the NHS. However, they also reported proactive care on the onset of COVID-19, whereby a variety of healthcare professionals would telephone to enquire how the person living with dementia and their family were managing, although this was not the case for all participants.

Some studies reported that few care services had adapted by providing remote support during the early stages of the pandemic. However, even when remote support was provided, carers felt it was not a direct replacement for the in-person contact and care that was offered and utilised pre-pandemic (Giebel et al., 2021d; Sriram et al., 2021). Similar issues were reported for remote healthcare consultations, which were often organised and handled by the carer (Tuijt et al., 2021b). There were also reports of digital barriers or exclusion from accessing remote healthcare and support services (e.g., Giebel et al., 2021d; Tuijt et al., 2021b).

\section{Impact on carer burden}

Twenty-eight studies documented the impacts of the COVID-19 pandemic on carer burden, with many noting increases in caring responsibilities or time dedicated to care (e.g, Borges-Machardo et al., 2020; Tam et al., 2021; Tsapanou et al., 2021). A range of tools were used to measure carer burden in quantitative studies ( $n=20)$; however, most relied on self-reports rather than validated measures (e.g., Boutoleau-Bretonniere et al., 2020; Cohen et al., 2021; Helvaci Yilmaz et al., 2021; Tsapanou et al., 2021). Two studies utilised the Zarit Burden Interview (Borg et al., 2021; Borelli et al., 2021). These studies reported high levels of burden, with Borg et al. (2021) noting 32.4\% of carers' scores indicated severe burden. Two studies used the Caregiver Burden Inventory (CBI; Altieri \& Santangelo, 2020; Penerai et al., 2020). In a pre-post study on the impact of confinement during the pandemic on carers in Italy, Penerai et al (2020) found statistically significant changes in CBI scores pre- and during lockdown, with an increase of approximately $10 \%$ of carers at risk for burning out. Large 
effect sizes were reported for total CBI scores and physical burden, and a medium effect size in time dependence and developmental burden. One study used the Care-related Quality of Life Instrument, reporting significant increases in subjective burden (Borges-Machado et al., 2020).

Impacts on carer burden have been associated with stage of dementia across quantitative studies, with Cohen et al. (2021a) noting levels of carer burden being particularly high for carers of people with advanced dementia after four weeks of quarantine. Significant differences in the influence of diagnostic type of dementia on levels of burden have not been observed (Altieri et al., 2021; Boutoleau-Betonniere et al., 2020). However, Boutoleau-Betonniere et al. (2020) did report that increases in burden occurred among carers of people living with behavioural variant frontotemporal dementia regardless of changes in neuropsychiatric symptoms, whereas increases in burden among carers of people living with Alzheimer's disease was related to changes in neuropsychiatric symptoms.

Findings from qualitative $(n=5)$ and mixed-methods $(n=1)$ studies show that carers attributed increases in burden to the suspension of care services, a lack of usual support from other family members, heightened feelings of responsibility and a need to take extra precautions to avoid infection (Rising et al., 2021; Sririam et al., 2021; Tuijt et al., 2021; Vaitheswaran et al., 2020; Rajagopalan et al., 2021; West et al., 2021). Some quantitative studies reported that carer burden was amplified by changes in the care needs of the person living with dementia due to symptom advancement during lockdown periods (see Part I). In Brazil, Borelli et al. (2021) found carers of people living with dementia whose cognition had worsened since March 2020 reporting significantly increased burden.

The ongoing nature of the COVID-19 pandemic and possibility of future lockdown periods have implications for carer burden. In Greece, Tsapanou et al. (2021) reported high levels of physical and psychological burden, which increased significantly from an initial lockdown to a second prolonged lockdown period.

\section{Impact on well-being}

Twenty-six studies reported on the impact of the pandemic of the pandemic on the wellbeing of carers, focusing specifically upon mental well-being and social wellbeing.

\section{Impact on mental wellbeing}

All 26 studies described the negative impact of the pandemic on carers' mental well-being. In a survey in Italy, Rainero et al. (2020) found $45.9 \%$ of carers reported increases in anxiety and $18.6 \%$ reported increases in depression since quarantine. Similarly, in France, Borg et al. (2021) found half of carers exhibited poor mental health, including depression, anxiety, or self-reported stress. Studies typically focused upon anxiety, depression and stress among carers (e.g., Altieri \& Santangelo, 2021; Carpinelli \& Mazzi et al. 2020, Hwang et al., 2021; Rainero et al., 2020; Zucca et al., 2021); however, some studies also reported negative effects upon sleep and eating disorders during home confinement (e.g., Carcavilla et al., 2021; Cohen et al., 2020). Validated measures of depression and anxiety included the GAD-7 (Alexopoulu et al., 2021; Hwang et al., 2021; Pongan et al., 2021), Italian version of the Hospital Anxiety and Depression Scale (HADS; Altieri \& Santangelo, 2021), the Center for Epidemiologic Studies Depression Scale (Hwang et al., 2021), Zung's depression and anxiety assessment scales (ZDAAS; Carpinelli Mazzi et al., 2020). Stress was primarily measured using the Perceived Stress Scale (Carpinelli Mazzi et al., 2020; Rusowicz et al., 2021).

The negative impact of the pandemic on carer's wellbeing and mental health was also noticed in qualitative ( $n=4)$ and mixed-methods studies ( $n=1)$. Many studies reported on the increased anxiety, fear, depression or stress levels (eg., Sriram et al., 2021; Rising et al., 2021; West et al., 2021; Rajagopalan et al., 2021). Most commonly the anxiety/fear was related to becoming infected with COVID-19 and/or infecting of a person with dementia (e.g., Sriram et al., 2021; Rising et al., 2021). The increased anxiety of carers was related to managing following the restrictions by person with dementia or other member of family/society (e.g., Sriram et al., 2021); worry about the condition of the person with dementia after pandemic (Giebel et al., 2021d) or ongoing fear for future (Hanna et al., 2021). In the UK, some carers have reported feeling of being strained and losing their freedom (West et al., 2021) or loss of hope (Hanna et al., 2021) and loss of control feelings (Giebel et al., 2021d). Carers from India have been also reporting increased negative feelings, including feeling lost (Rajagopalan et al., 2021). Carers in the USA reported putting their own needs on hold due to the increased demands of caregiving (Rising et al., 2021).

Several studies have also reported on coping with providing care during the pandemic, used strategies and self-protective factors. Losada et al. (2021) study presented that majority of carers considered that they coped well whereas Salva et al. (2021) reported that active coping strategies were used by $57 \%$ of carers. The frequently used coping strategies were related to self-care or described, i.e., by taking up some new or creative activities (e.g., Tulloch et al, 2021; West et al., 2021); reading, doing house chores (e.g., Rising et al., 2021), maintaining social connections or being able to get out (Tuijt et al., 2021). Factors including effective communication during pandemic, existing support networks, coping mechanisms and lifestyle factors including exercises or access to green spaces were reported as factors contributing to resilience of carers by Hanna et al. (2022).

A few studies focussed on positive aspects of providing care during the pandemic. In an Australian study, carers noted that providing care during the pandemic revealed their inner strength as well as their priorities and values having changed, whilst the relationship between them and care recipients deepened (Tulloch et al., 2021). Being close and spending more time with loved ones was also presented in other studies (Rising et al., 2021; Sriram et al., 2021). Similarly, some carers in the Losada et al. (2021) study reported an increase in positive emotions such as hope and happiness during the pandemic. The pandemic has also offered an opportunity to rest to some carers (West et al., 2021).

\section{Impact on social health}

The impact of the pandemic on social health was reported in six studies. These studies reported enhanced levels of social isolation and loneliness among carers during the pandemic. In a survey of 395 carers in Canada, Tam et al. (2021) found that most carers felt isolated, left out, and lacking companionship some of the time or often, with $89 \%$ also feeling more or somewhat more isolated since the pandemic. A sense of isolation and abandonment was also 
reported in an Italian study, but less frequently than other symptoms of stress (Zucca et al., 2021). There was a notable lack of studies using validated measures of social health.

Social isolation and loneliness were also documented in qualitative studies. In the USA, participants reported that the inability to socialise with their community, travel for leisure, and see family members were primary issues (Rising et al., 2021). In an interview study in the UK, carers expressed feelings of loneliness during a period of lockdown which they attributed to their main social contact being the person living with dementia whom they cared for (Hanna et al., 2021). Research with carers from minority ethnic backgrounds in the UK indicated that social interaction was also widely reduced, often due to fears of contracting COVID-19 and transmitting it to loved ones (West et al., 2021). These carers also discussed being unable to attend communal culturally relevant events as a negative impact of the pandemic, such as church or temple.

In one study, the majority of carers reported turning to technology to connect with others (Tam et al., 2021). However, only $19 \%$ of carers reported that using technology to connect with others felt the same as interacting with them in-person.

\section{Quality ratings}

All but three studies were of good quality (see Tables 2 and 3), with scores ranging from 0.65 to 1.00 .

\section{Discussion}

This appears to be the first comprehensive systematic review on the early impacts of the pandemic on unpaid dementia carers. Whilst a previous systematic review has explored the impacts on health only (Hughes et al., 2021), this review purposefully synthesised the evidence into different aspects of the lives of unpaid carers, including mental health and well-being, as well as access to care for themselves and their relatives, and the impacts this in turn had on their health and mental health. Substantial evidence generated in the early stages of the pandemic has shown the negative effects of the pandemic on accessing dementia-related care and support (Dassel et al., 2021; Werner et al., 2021), which led to increased caring duties for family members and friends (Giebel et al., 2020), subsequently perniciously affecting carers' mental health and well-being (Carpinelli Mazzi et al., 2020; Tam et al., 2021).

Accessing care and support is not only vital for the person living with the condition, but also for the unpaid carer - both of whom have experienced increased barriers in doing so since the pandemic (also see Part I). The majority of research has highlighted social care and social support reductions since the pandemic, primarily focusing on community care, with some research indicating early care home access issues (Giebel et al., 2021c) and health care utilisation barriers (Tuijt et al., 2021). Being unable to access care, either for themselves via peer support groups for example, or predominantly for their relative with dementia to gain some temporary respite from caring duties (Tretteteig et al., 2017), has wide-ranging implications for the carer. Without any respite or time off from caring, carers have been found to be more likely to be burned out (Cohen et al., 2021b). Whilst evidence reported in this review has already highlighted increased burden for many early on, the continuing nature of the pandemic is likely having long-term repercussions on carers leading to burnout. Future research needs to follow up carers and explore the long-term effects, however, early evidence from this review strongly indicates a greater need to adequately and equitably support unpaid carers in their roles, and as individuals themselves.

These effects have not only been noted regarding carer burden, but also more widely for mental well-being. All studies but one (Tulluoch et al., 2021) noted at least some aspects of negative impacts on the mental and social health of unpaid carers, particularly focusing on depression, anxiety, and stress, as well as social isolation and loneliness. Tulloch et al. (2021) purposefully only analysed positive experiences however, clearly producing biased findings. For many carers, engaging with the person with dementia was their only point of face-to-face social contact during the pandemic, generating feelings of loneliness (Hanna et al., 2021; Tam et al., 2021). This can particularly be the case for older spousal carers living with their relative with dementia, which can add to potential feelings of burnout and lack of respite from their caring duties. Considering the wider restrictions impacting on everyone, across the globe, it is unclear to what extent heightened levels of caring duties and lack of respite from caring contributed to poorer mental well-being, and to what extent generally living through an unknown and novel pandemic contributed. Longitudinal survey data has evidenced reductions in mental health across the general UK population in the early stages of the pandemic compared to prior (Pierce et al., 2020). On top of these impacts, evidence from this review illustrates the significant impact that informal caring duty and formal care access changes have had on unpaid carers (Dassel et al., 2021; Rajagopalan et al., 2021;). Whilst more research is needed on the long-lasting mental health needs of unpaid dementia carers, and unpaid carers in general, findings from this review clearly highlight a need for improved access to mental health support, something that should be in place anyways considering the gravity of experiencing the diagnosis of a loved one and living through the diagnosis providing care and support.

One way for carers to try and stay connected with people other than the person with dementia was via digital technology. Whilst care services were not adapted to provide digital support at the beginning of the pandemic, some services very slowly adapted to providing care remotely, particularly peer support groups (i.e., Giebel et al., 2021c). Connecting with peers remotely to bypass the growing sense of social isolation and loneliness was also a solution for carers as reported by Tam et al. (2021), although less than a fifth of carers felt that digital social connectivity was as beneficial as face-to-face contact and a number of barriers have been identified. Recent research has highlighted how carer age also matters when connecting digitally with dementia services during the pandemic, as younger carers (adult children) appeared to be better equipped, highlighting the digital divide (Arighi et al., 2021). Whilst more research is required into the digital dementia care experiences since the pandemic, emerging research illustrates how digital connectivity can provide some benefits when lacking face-to-face engagement. Even when connecting remotely with peers and services, the unpaid carer still has to be with the person with dementia though to access care, and cannot engage in their own activities. Therefore, care should be provided face-to-face again in safe formats as soon as possible, whilst some long-term benefits may be drawn from digitally adapted services for people living in more rural communities - a frequent previous barrier to engaging with support services (Innes et al., 2006). 
The mix of lack of support and access to care, poor mental well-being, and increased carer burden are all likely to contribute to people with dementia entering a care home earlier, at least compared to non-pandemic circumstances. In pre-pandemic times, when unpaid carers were unable to care for their relative at home any longer, due to increased carer burden or too many care needs, people with dementia would normally enter a care home (Sutcliffe et al., 2017). In the best case scenario, this would have been planned in advance to provide care home entry at the right time. Since the pandemic, people with dementia also appear to have deteriorated faster and received less care support in the community, as Part I of this interlinked systematic review has shown (Giebel et al., submitted) has shown, confirming earlier reported results (Suarez-Gonzalez et al., 2021). Thus, it is likely that people with dementia have entered a care home faster since the pandemic, based on the negative impacts of COVID-19 on community-residing people with dementia and carers, as evidenced in this review. However, care homes have faced different levels of restrictions, which are ongoing, often not allowing unpaid carers to visit their relative (Backhaus et al., 2021) and making it difficult to plan in advance. This may lead to further increases in carer burden, as well as guilt, if carers are unable to care for their relative at home but delay care home entry due to those restrictions, whilst others may see no alternative but to have their relative admitted. Very early indications about faster care home entry, and the ensuing guilt experienced by unpaid carers, has been reported in one of the included studies here in summer 2020 in the UK for example (Giebel et al., 2021c). However, more long-term research is required after two years of pandemic restrictions to explore the impact on carer wellbeing and burden on care home entry during the pandemic.

\section{Limitations}

This mixed-method systematic review benefitted from searching numerous data bases and producing a timely report of the impact of the pandemic on unpaid carers. However, due to the nature of the research and a continuously growing evidence base, it was not feasible to update the searches. This review already included a large number of studies after exclusion criteria were applied, and thus provides a timely and time-period specific synthesis of the evidence base to inform future research and policy making. In terms of the research included, some studies were limited in providing retrospective accounts of for example mental health prior to the pandemic and changes noticed, as opposed to pre and post assessments using validated measures. However, mental health or burden is in general assessed largely by asking the person affected. The recall bias needs to be taken into account. However, a state-of the art pre-pandemic assessment was not presented for obvious reasons. Therefore, these data provide suitable evidence. One wider limitation, but also advantage, of this review is that it considered studies from 18 countries. Restrictions differed between countries and even between regions making it difficult to relate a type or severity of measure to an outcome. However, even considering this heterogeneity the studies identified a comparable impact on careers. All reported negative impacts on the support system, well-being and mental health of unpaid carers (except one study which purposefully explored positive experiences from interviews and was therefore biased - Tulloch et al., 2021). However, there were limitations in findings from lower- and middle-income countries (LMICs), with only two studies reporting on India and Brazil (Azavedo et al., 2021; Rajagopalan et al., 2021). Impacts are likely to differ across high-impact countries and LMICs, indicating a need for more research into the impacts in LMICs.

\section{Conclusions}

Unpaid carers, similar to people with dementia, have been harmfully affected during the pandemic from access to adequate care and support to increased levels of carer burden and poor mental health. Findings in this review from across the globe thus illustrate how unpaid carers urgently need to be supported better in not only their caring role, but also as an individual, taking into account their own personal needs. Whilst restrictions may ease in certain countries and many, albeit not all, societies benefit from protection offered from vaccinations, the early impacts of the pandemic are likely going to have long-lasting effects on the mental and physical health of unpaid carers. Future research ought to explore the long-lasting impacts of COVID-19 on this group, especially in light of care recipients entering care homes.

\section{Declarations}

\section{Acknowledgements}

We would like to acknowledge the support of Adel Afin in running and documenting the literature search.

\section{Funding}

This is independent research funded by the National Institute for Health Research Applied Research Collaboration North West Coast (ARC NWC). The views expressed in this publication are those of the author(s) and not necessarily those of the National Institute for Health Research or the Department of Health and Social Care. This review was also supported by a grant from the Geoffrey and Pauline Martin Trust.

\section{Conflicts of interest}

None.

\section{Author contributions}

CG led the systematic review, drafted the protocol and manuscript, and scored citations for Stage 1 and 2. ASG generated the search strategy. RT, KL, CT, KHL, EW scored citations for inclusion in the review. EW extracted all data from included studies and quality rated all studies. RT, CT, ASG, KHL, KL, EW, JC, HT discussed the findings jointly, placed them into context, and read through drafts of the manuscripts before approving the final draft.

\section{References}


1. Alexopoulos, P., Soldatos, R., Kontogianni, E., et al. (2021). COVID-19 Crisis Effects on Caregiver Distress in Neurocognitive Disorder. J Alzheimers Dis, $79(1), 459-466$.

2. Altieri, M., \& Santangelo, G. (2021). The Psychological Impact of COVID-19 Pandemic and Lockdown on Caregivers of People With Dementia. Am J Geriatr Psychiatry, 29(1), 27-34.

3. Arighi A, Fumagalli GG, Carandini T, et al. Facing the digital divide into a dementia clinic during COVID-19 pandemic: caregiver age matters. Neurological Sciences 2021;42:1247-1251.

4. Azevedo, L., Calandri, I. L., Slachevsky, A., Graviotto, H. G., Vieira, M. C. S., Andrade, C. B.,.. . Caramelli, P. (2021). Impact of Social Isolation on People with Dementia and Their Family Caregivers. J Alzheimers Dis, 81(2), 607-617.

5. Bacsu JDR, O'Connell ME, Webster C, et al. A scoping review of COVID-19 experiences of people living with dementia. Canadian Journal of Public Health 2021;112:400-411.

6. Bianchetti A, Rozzini R, Guerini F, et al. Clinical presentation of COVID-19 in dementia patients. Journal of Nutrition, Health \& Ageing 2020; doi: $10.1007 /$ s12603-020-1389-1

7. Borelli, W. V., Augustin, M. C., de Oliveira, P. B. F., Reggiani, L. C., Bandeira-de-Mello, R. G., Schumacher-Schuh, A. F.,.. . Castilhos, R. M. (2021). Neuropsychiatric Symptoms in Patients with Dementia Associated with Increased Psychological Distress in Caregivers During the COVID-19 Pandemic. J Alzheimers Dis, 80(4), 1705-1712.

8. Borg, C., Rouch, I., Pongan, E., Getenet, J. C., Bachelet, R., Herrmann, M.,.. . Dorey, J. M. (2021). Mental Health of People with Dementia During COVID-19 Pandemic: What Have We Learned from the First Wave? J Alzheimers Dis, 82(4), 1531-1541.

9. Borges-Machado, F., Barros, D., Ribeiro, O., \& Carvalho, J. (2020). The Effects of COVID-19 Home Confinement in Dementia Care: Physical and Cognitive Decline, Severe Neuropsychiatric Symptoms and Increased Caregiving Burden. Am J Alzheimers Dis Other Demen, 35, 1533317520976720.

10. Boutoleau-Bretonniere, C., Pouclet-Courtemanche, H., Gillet, A., Bernard, A., Deruet, A. L., Gouraud, I.,.. . El Haj, M. (2020). Impact of Confinement on the Burden of Caregivers of Patients with the Behavioral Variant of Frontotemporal Dementia and Alzheimer Disease during the COVID-19 Crisis in France. Dement Geriatr Cogn Dis Extra, 10(3), 127-134.

11. Carcavilla, N., Pozo, A. S., Gonzalez, B., Moral-Cuesta, D., Roldan, J. J., Erice, V., \& Remirez, A. G. (2021). Needs of Dementia Family Caregivers in Spain During the COVID-19 Pandemic. J Alzheimers Dis, 80(2), 533-537.

12. Carpinelli Mazzi, M., lavarone, A., Musella, C., De Luca, M., de Vita, D., Branciforte, S.,.. . Postiglione, A. (2020). Time of isolation, education and gender influence the psychological outcome during COVID-19 lockdown in caregivers of patients with dementia. Eur Geriatr Med, 11(6), $1095-1098$. doi:10.1007/s41999-020-00413-z

13. Clemmensen TH, Lauridsen HH, Andersen-Ranberg K, Kristensen HK. 'I know his needs better than my own' - carers' support needs when caring for a person with dementia. Scandinavian Journal of Caring Sciences 2021;35(2):586-599.

14. Cohen, G., Russo, M. J., Campos, J. A., \& Allegri, R. F. (2020a). COVID-19 Epidemic in Argentina: Worsening of Behavioral Symptoms in Elderly Subjects With Dementia Living in the Community. Front Psychiatry, 11, 866. doi:10.3389/fpsyt.2020.00866

15. Cohen, G., Russo, M. J., Campos, J. A., \& Allegri, R. F. (2020b). Living with dementia: increased level of caregiver stress in times of COVID-19. Int Psychogeriatr, 32(11), 1377-1381. doi:10.1017/S1041610220001593

16. Dassel, K. B., Towsley, G. L., Utz, R. L., Ellington, L., Terrill, A., Scammon, D.,.. . Mickens, M. (2021). A Limited Opportunity: CoVID-19 and Promotion of Advance Care Planning. Palliat Med Rep, 2(1), 194-198.

17. Giebel C, Hanna K, Rajagopal M, et al. The potential dangers of not understanding COVID-19 public health restrictions in dementia: "It's a groundhog day - every single day she does not understand why she can't go for a walk". BMC Public Health 2021;21:762.

18. Giebel, C., Hanna, K., Cannon, J., Eley, R., Tetlow, H., Gaughan, A.,.. . Gabbay, M. (2020). Decision-making for receiving paid home care for dementia in the time of COVID-19: a qualitative study. BMC Geriatr, 20(1), 333.

19. Giebel, C., Pulford, D., Cooper, C., Lord, K., Shenton, J., Cannon, J.,.. . Gabbay, M. (2021b). COVID-19-related social support service closures and mental wellbeing in older adults and those affected by dementia: a UK longitudinal survey. BMJ Open, 11(1), e045889.

20. Giebel, C., Hanna, K., Callaghan, S., Cannon, J., Butchard, S., Shenton, J.,.. . Gabbay, M. (2021c). Navigating the new normal: accessing community and institutionalised care for dementia during COVID-19. Aging Ment Health, 1-6. doi:10.1080/13607863.2021.1914545

21. Giebel, C., Cannon, J., Hanna, K., Butchard, S., Eley, R., Gaughan, A.,.. . Gabbay, M. (2021d). Impact of COVID-19 related social support service closures on people with dementia and unpaid carers: a qualitative study. Aging Ment Health, 25(7), 1281-1288.

22. Giebel, C., Sutcliffe, C., Darlington-Pollock, F., Green, M. A., Akpan, A., Dickinson, J.,.. . Gabbay, M. (2021e). Health Inequities in the Care Pathways for People Living with Young- and Late-Onset Dementia: From Pre-COVID-19 to Early Pandemic. Int J Environ Res Public Health, 18(2).

23. Giebel, C., Lord, K., Cooper, C., Shenton, J., Cannon, J., Pulford, D.,.. . Gabbay, M. (2021f). A UK survey of COVID-19 related social support closures and their effects on older people, people with dementia, and carers. Int J Geriatr Psychiatry, 36(3), 393-402.

24. Hanna, K., Giebel, C., Butchard, S., Tetlow, H., Ward, K., Shenton, J.,.. . Gabbay, M. (2022). Resilience and supporting people living with dementia during the time of COVID-19; A qualitative study. Dementia (London), 21(1), 250-269.

25. Hanna, K., Giebel, C., Tetlow, H., Ward, K., Shenton, J., Cannon, J.,.. . Gabbay, M. (2021). Emotional and Mental Wellbeing Following CoVID-19 Public Health Measures on People Living With Dementia and Carers. J Geriatr Psychiatry Neurol, doi:10.1177/0891988721996816

26. Hariyanto TI, Putri C, Arisa J, et al. Dementia and outcomes from coronavirus disease 2019 (COVID-19) pneumonia: A systematic review and metaanalysis. Archives of Gerontology and Geriatrics 2021;93:104299.

Page $17 / 19$ 
27. Helvaci Yilmaz, N., Polat, B., Ermis, A., \& Hanoglu, L. (2021). Clinical deterioration of Alzheimer's disease patients during the Covid-19 pandemic and caregiver burden. Journal of experiemental and clinical medicine, 38(3), 255-259.

28. Hughes MC, Liu Y, Baumbach A. Impact of COVID-19 on the health and well-being of informal caregivers of people with dementia: a rapid systematic review. Gerontology and Geriatric Medicine 2021; doi: 10.1177/2F23337214211020164

29. Hwang, Y., Connell, L. M., Rajpara, A. R., \& Hodgson, N. A. (2021). Impact of COVID-19 on Dementia Caregivers and Factors Associated With their Anxiety Symptoms. Am J Alzheimers Dis Other Demen, 36, 15333175211008768.

30. Innes A, Cox S, Smith A, et al. Service provision for people with dementia in rural Scotland: Difficulties and innovations. Dementia 2006;5(2):249-270.

31. Kmet LM, Lee RC, Cook LS. Standard quality assessment criteria for evaluating primary research papers from a variety of fields. Edmonton: Alberta Heritage Foundation for Medical Research. AHFMR, 2004.

32. Kostyál, L. Á., Széman, Z., Almási, V. E., Fabbietti, P., Quattrini, S., Socci, M.,.. . Gagliardi, C. (2021). Impact of the COVID-19 Pandemic on Family Carers of Older People Living with Dementia in Italy and Hungary. Sustainability, 13(13).

33. Losada, A., Vara-Garcia, C., Romero-Moreno, R., Barrera-Caballero, S., Pedroso-Chaparro, M. D. S., Jimenez-Gonzalo, L.,.. . Marquez-Gonzalez, M. (2021). Caring for Relatives with Dementia in Times of COVID-19: Impact on Caregivers and Care-recipients. Clin Gerontol, 1-15. doi:10.1080/07317115.2021.1928356

34. Ng, K. P., Chiew, H. J., Hameed, S., Ting, S. K. S., Ng, A., Soo, S. A.,.. . Kandiah, N. (2020). Frontotemporal dementia and COVID-19: Hypothesis generation and roadmap for future research. Alzheimers Dement (N Y), 6(1), e12085.

35. Orellana K, Manthorpe J, Tinker A. Day centres for older people - attendance characteristics, access routes and outcomes of regular attendance: findings of exploratory mixed methods case study research. BMC Geriatrics 2020;20:158.

36. Parveen S, Peltier C, Oyebode JR. Perceptions of dementia and use of services in minority ethnic communities: a scoping exercise. Health and Social Care in the Community 2017;25(2):734-742.

37. Pierce M, Hope H, Ford T, et al. Mental health before and during the COVID-19 pandemic: a longitudinal probability sample survey of the UK population. The Lancet Psychiatry 2020;10(7):883-892.

38. Panerai, S., Tasca, D., Musso, S., Catania, V., Ruggeri, F., Raggi, A.,.. . Ferri, R. (2016). The impact of COVID-19 confinement on the neurobehavioral manifestations of people with Major Neurocognitive Disorder and on the level of burden of their caregivers. Life Span and Disability(2), 303-320. Retrieved from http://www.lifespanjournal.it/client/abstract/ENG371_6.\%20Panerai.pdf

39. Pongan, E., Dorey, J. M., Borg, C., Getenet, J. C., Bachelet, R., Lourioux, C.,.. . Rouch, I. (2021). COVID-19: Association Between Increase of Behavioral and Psychological Symptoms of Dementia During Lockdown and Caregivers' Poor Mental Health. J Alzheimers Dis, 80(4), $1713-1721$.

40. Rainero, I., Bruni, A. C., Marra, C., Cagnin, A., Bonanni, L., Cupidi, C.,.. . Group, S. I. C.-S. (2020). The Impact of COVID-19 Quarantine on Patients With Dementia and Family Caregivers: A Nation-Wide Survey. Front Aging Neurosci, 12, 625781.

41. Rajagopalan, J., Arshad, F., Hoskeri, R. M., Nair, V. S., Hurzuk, S., Annam, H.,.. . Alladi, S. (2022). Experiences of people with dementia and their caregivers during the COVID-19 pandemic in India: A mixed-methods study. Dementia (London), 21(1), 214-235.

42. Rising, K. L., Salcedo, V. J., Amadio, G., Casten, R., Chang, A., Gentsch, A.,.. . Gerolamo, A. M. (2022). Living Through the Pandemic: The Voices of Persons With Dementia and Their Caregivers. J Appl Gerontol, 41(1), 30-35.

43. Rusowicz, J., Pezdek, K., \& Szczepanska-Gieracha, J. (2021). Needs of Alzheimer's Charges' Caregivers in Poland in the Covid-19 Pandemic-An Observational Study. Int J Environ Res Public Health, 18(9). doi:10.3390/ijerph18094493

44. Savla, J., Roberto, K. A., Blieszner, R., McCann, B. R., Hoyt, E., \& Knight, A. L. (2021). Dementia Caregiving During the "Stay-at-Home" Phase of COVID-19 Pandemic. J Gerontol B Psychol Sci Soc Sci, 76(4), e241-e245.

45. Sriram, V., Jenkinson, C., \& Peters, M. (2021). Impact of COVID-19 restrictions on carers of persons with dementia in the UK: a qualitative study. Age Ageing, 50(6), 1876-1885.

46. Suarez-Gonzalez A, Rajagopalan J, Livingston G, Alladi S. The effect of COVID-19 isolation measures on the cognition and mental health of people living with dementia: A rapid systematic review of one year of quantitative evidence. EClinical Medicine 2021;39:101047.

47. Talbot, C. V., \& Briggs, P. (2021). 'Getting back to normality seems as big of a step as going into lockdown': the impact of the COVID-19 pandemic on people with early to middle stage dementia. Age Ageing, 50(3), 657-663.

48. Tam, M. T., Dosso, J. A., \& Robillard, J. M. (2021). The Impact of a Global Pandemic on People Living with Dementia and Their Care Partners: Analysis of 417 Lived Experience Reports. J Alzheimers Dis, 80(2), 865-875.

49. Tsapanou, A., Zoi, P., Kalligerou, F., Blekou, P., \& Sakka, P. (2021). The Effect of Prolonged Lockdown Due to COVID-19 on Greek Demented Patients of Different Stages and on Their Caregivers. J Alzheimers Dis, 83(2), 907-913. doi:10.3233/JAD-210702

50. Tretteteig S, Vatne S, Rokstad AMM. The influence of day care centres designed for people with dementia on family caregivers - a qualitative study. BMC Geriatrics 2017;17.

51. Tuijt, R., Frost, R., Wilcock, J., Robinson, L., Manthorpe, J., Rait, G., \& Walters, K. (2021a). Life under lockdown and social restrictions - the experiences of people living with dementia and their carers during the COVID-19 pandemic in England. BMC Geriatr, 21(1), 301.

52. Tuijt, R., Rait, G., Frost, R., Wilcock, J., Manthorpe, J., \& Walters, K. (2021b). Remote primary care consultations for people living with dementia during the COVID-19 pandemic: experiences of people living with dementia and their carers. Br J Gen Pract, 71(709), e574-e582.

53. Tulloch, K., McCaul, T., \& Scott, T. L. (2021). Positive Aspects of Dementia Caregiving During the COVID-19 Pandemic. Clin Gerontol, 1-11. doi:10.1080/07317115.2021.1929630 
54. Vaitheswaran, S., Lakshminarayanan, M., Ramanujam, V., Sargunan, S., \& Venkatesan, S. (2020). Experiences and Needs of Caregivers of Persons With Dementia in India During the COVID-19 Pandemic-A Qualitative Study. Am J Geriatr Psychiatry, 28(11), 1185-1194.

55. Werner, P., Tur-Sinai, A., \& AboJabel, H. (2021). Examining Dementia Family Caregivers' Forgone Care for General Practitioners and Medical Specialists during a COVID-19 Lockdown. Int J Environ Res Public Health, 18(7).

56. West, E., Nair, P., Barrado-Martin, Y., Walters, K. R., Kupeli, N., Sampson, E. L., \& Davies, N. (2021). Exploration of the impact of the COVID-19 pandemic on people with dementia and carers from black and minority ethnic groups. BMJ Open, 11(5), e050066.

57. World Health Organisation (WHO). Dementia - Factsheet. Geneva, 2021. Last accessed 27/01/2022: https://www.who.int/news-room/factsheets/detail/dementia

58. Zucca, M., Isella, V., Lorenzo, R. D., et al. (2021). Being the Family Caregiver of a Patient With Dementia During the Coronavirus Disease 2019 Lockdown. Front Aging Neurosci, 13, 653533. doi:10.3389/fnagi.2021.653533

59. Zuin M, Guasti P, Roncon L, Cervellati C, Zuliani G. Dementia and the risk of death in elderly patients with COVID-19 infection: Systematic review and metaanalysis. International Journal of Geriatric Psychiatry 2020;36(5):697-703.

\section{Figures}

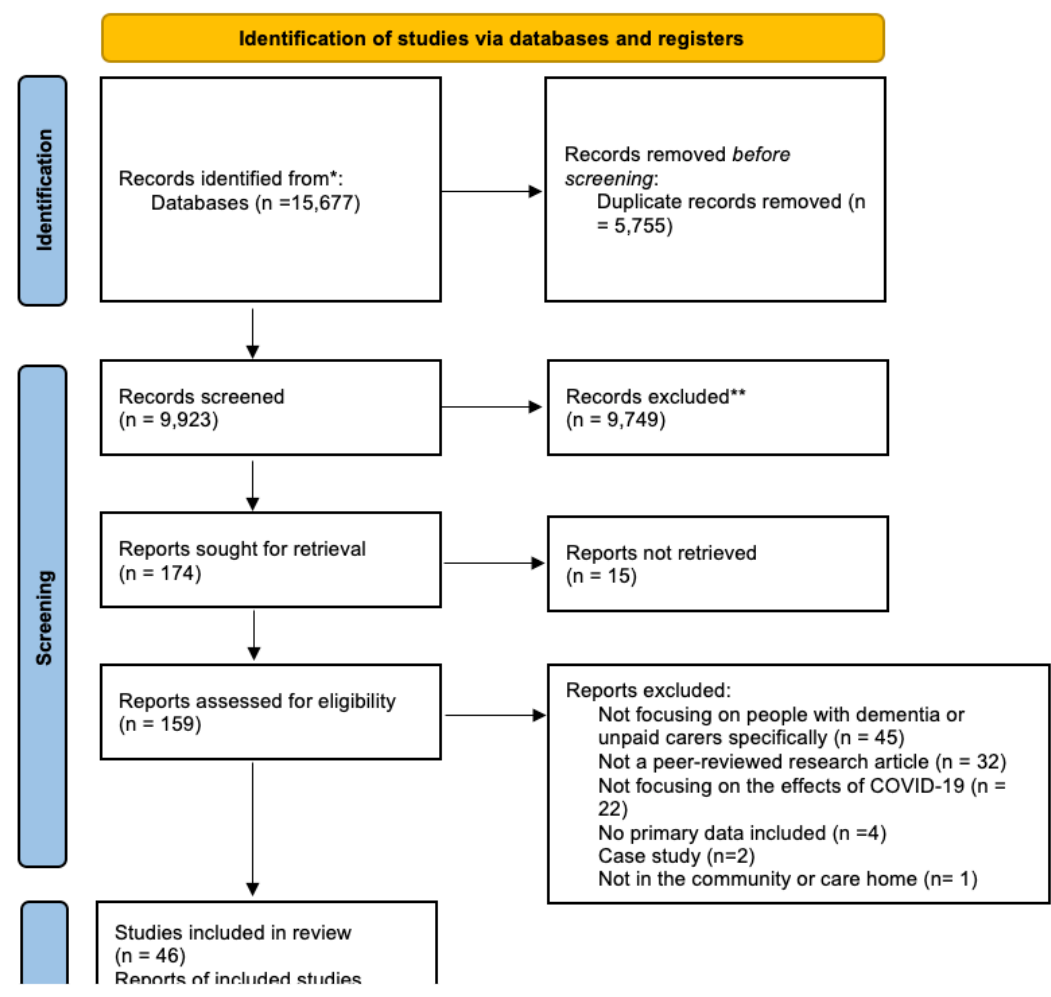

Figure 1

PRISMA Flowchart 